\title{
Ad Foolen
}

\section{Ich denk, mich tritt ein Pferd! \\ Phraseologische Ausdrücke der Überraschung im modernen Deutsch}

\section{Das Mich-tritt-ein-Pferd-Paradigma}

Duden 11 (2002) enthält einige Redewendungen, die den Ausgangspunkt dieses Beitrags bilden. Ich gebe die Einträge in (1-4) vollständig wieder:

(1) Ich denk, mich tritt ein Pferd (salopp). Ausdruck der Verblüffung. Ich dachte, mich tritt ein Pferd. Das war Mutter Wibeau (Ulrich Plenzdorf, Die neuen Leiden des jungen W., 1973). (ebd.: 574)

(2) Ich denk, mich küsst/knutscht ein Elch (ugs. scherzh.). Ausdruck äußerster Überraschung. Ich denk, mich küsst ein Elch - steht doch plötzlich mein alter Freund Willi neben mir! Die Redensart ist eine Abwandlung von ,ich denk, mich tritt ein Pferd“. Das absurde Bild soll das völlig Überraschende einer Situation noch stärker veranschaulichen. (ebd.: 189)

(3) [Ich denk,] mich laust der Affe! (salopp). Ich bin völlig verblüfft! Er kniff das linke Auge zu, grinste und sagte: „Mich laust der Affe! Du bist doch der Abi!“ (Hörzu 1980). (ebd.: 36)

(4) Ich denk, ich steh im Wald (ugs.). Ausdruck der Verwunderung, Entrüstung. Schnappt der Kerl sich doch, ohne zu fragen, mein Fahrrad - ich denk, ich steh im Wald! (ebd.: 838)

Duden 11 verweist bei den verschiedenen Einträgen nicht auf die anderen drei, außer bei (2), das als Abwandlung von (1) bezeichnet wird. Die vier Ausdrücke sind verwandt, sie ähneln sich in der Form und können in derselben Situation für denselben Zweck benutzt werden, nämlich zum Ausdruck der Überraschung bei Konfrontation mit einer unerwarteten Situation. In den Belegen $\mathrm{zu}$ den Wendungen (1-3) geht es um die plötzliche Konfrontation mit der Anwesenheit, Identität oder Handlung einer anderen Person. Wie in den Belegen zu sehen ist, wird die unerwartete Situation im Text selber auch spezifiziert, entweder nach der Wendung, wie in (1-3), oder davor, wie in (4). Es handelt sich also eigentlich um eine satzübergreifende, textuelle „Konstruktion“, die aus zwei Teilen besteht, der Wendung selbst 
und dem „Anlass“, der zum Ausruf in Form einer Wendung führt. Die Wendung selbst besteht immer aus einem Matrixsatz in der 1. Person Singular, denk als Matrixverb, und einem Nebensatz in SVO-Wortstellung. Stilistisch werden die Wendungen in Duden 11 als umgangssprachlich, salopp, scherzhaft charakterisiert.

Es sieht also so aus, als handle es sich hier um ein (kleines) Paradigma von Wendungen, die in Form und Funktion Familienähnlichkeit aufweisen. Vereinfachend werde ich im Weiteren von dem Mich-tritt-ein-Pferd-Paradigma sprechen.

In diesem Beitrag möchte ich vier Fragen näher betrachten:

(i) Seit wann werden die verschiedenen Wendungen benutzt?

(ii) Gibt es noch andere Wendungen als (1-4), die wir diesem Paradigma hinzufügen können?

(iii) Welche der genannten Merkmale sind obligatorisch, welche können weggelassen oder abgewandelt werden?

(iv) Können wir die Bedeutung/Funktion, die den Wendungen in Duden 11 zugesprochen wird, genauer charakterisieren? Insbesondere möchte ich erkunden, ob es sinnvoll wäre, das Konzept der Mirativität (DeLancey 1997) ins Spiel zu bringen.

Diesen Fragen sind jeweils die Abschnitte 2, 3, 4 und 5 dieses Beitrags gewidmet. Zur Illustration werde ich Beispiele aus dem Internet (u.a. Twitter) heranziehen. Als nützliche Quelle stellte sich der im Internet verfügbare Redensarten-Index heraus, ${ }^{1}$ den ich weiter abkürzend als Redensarten andeuten werde.

\section{Gebrauch und Bekanntheit der Wendungen}

2012 erschien eine Sammlung von Redewendungen, zusammengestellt von „Dr. Wort“, unter dem Titel Mich laust der Affe. Diese Titelwahl deutet darauf hin, dass der Autor diese Wendung als bekannt einschätzt. Können wir auch hinsichtlich der anderen Wendungen Hinweise für aktuellen Gebrauch oder wenigstens Bekanntheit finden?

Für einen ersten Eindruck habe ich ein Dutzend Muttersprachler verschiedenen Alters gefragt, ob sie die Wendungen (1-4) kennen. Die Antwort war meistens bestätigend, und sie waren auch im Stande, weitere Wendungen mit ähnlicher Bedeutung zu nennen. Das deutet darauf hin, dass es sich hier um ein Paradigma handelt, das zur (passiven) idiomatischen Kompetenz eines durchschnittlichen deutschen Muttersprachlers gehört.

1 www.redensarten-index.de/suche.php (Stand: 25.7.2017). 
Ein zweiter Hinweis findet sich in den Abfragestatistiken, die Redensarten bei den einzelnen Wendungen registrieren. Für die Wendungen (1-4) sind die monatlichen durchschnittlichen Abruffrequenzen seit Januar 2015 108, bzw. 38, 70 und 91. Die Wendungen werden also regelmäßig nachgeschlagen. Einerseits kann man das Richtung Unbekanntheit deuten, andererseits gibt es offensichtlich Nachschlagebedarf, was auf jeden Fall auf Interesse, vielleicht auch auf Gebrauchsintention hindeuten kann.

Die Bekanntheit und wahrscheinlich auch die Gebrauchsfrequenz der Wendungen wurde bestimmt auch dadurch verstärkt, dass sie in Fernsehserien, Filmen und von bekannten Personen benutzt wurden. In Redensarten wird beim Eintrag Ich denk, mich tritt ein Pferd erwähnt, dass die Wendung „wahrscheinlich aus der Fernsehserie Die Zwei (mit Roger Moore und Tony Curtis) [stammt] bzw. der dazugehörigen deutschen Übersetzung, die durch die große Popularität der Serie Eingang in den täglichen Sprachgebrauch gefunden hat“. Die Serie wurde in Großbritannien 1971 und im ZDF 1972 ausgestrahlt. ${ }^{2}$ An derselben Stelle in Redensarten wird bemerkt, dass ,die Redewendung [...] auch durch die Nutzung während eines Interviews durch den damaligen SPD-Bundesfinanzminister Hans Apel (1974-78) bekannt [wurde]“.

Ich denk mich trifft ein Pferd war auch der Titel eines deutschen Films aus dem Jahre 1975, mit Uschi Glas in der Hauptrolle. Im Wikipedia-Artikel zum Film wird auf die Verwendung der Wendung durch Apel hingewiesen: „Der Filmtitel basiert auf einem Ausspruch des einstigen Bundesfinanzministers Hans Apel, der diesen Ausdruck 1975 durch ein Interview mit dem Fernsehjournalisten Friedrich Nowottny landesweit bekannt und populär machte. “3

Bei der weiteren Suche fand ich noch einen anderen Filmtitel Ich glaub', mich tritt ein Pferd, also mit dem Verb glauben, aus dem Jahr 1978. Der englische Originaltitel lautet National Lampoon's Animal House. Die Abwandlung mit glauben war wohl nötig, weil der Titel mit denken schon drei Jahre vorher vergeben worden war. Dass die Wendung trotzdem erneut eingesetzt wurde, kann als Indiz dafür gewertet werden, dass die Wendung in den 1970er Jahren hohe Popularität genoss. Im Jahr 1981 bekam der englische Film Stripes in der deutschen Fassung den Titel Ich glaub', mich knutscht ein Elch! und 1982 erschien die Filmkomödie Ich glaub, ich steh im Wald. Es sieht fast so aus, als ob sich in den 1970er und 1980er Jahren vorübergehend ein eigenes Paradigma von Filmtiteln herausgebildet hat.

2 Man müsste im Originaltext nachschauen, welche englische Wendung Anlass für die deutsche war.

3 https://de.wikipedia.org/wiki/Ich_denk\%E2\%80\%99_mich_tritt_ein_Pferd (Stand: 23.11.2017). 


\section{Welche Wendungen gehören noch zum Paradigma?}

Während Duden 11 sich auf die Wendungen (1-4) beschränkt, finden sich in Redensarten weitere Ausdrücke, die zum Paradigma gerechnet werden können. Ich gebe die Einträge wörtlich wieder:

(5) Ich glaub, mein Schwein pfeift! Ausruf der Verwunderung/Verärgerung / Fassungslosigkeit/Überraschung/Empörung; Reaktion auf ein unerwartetes Ereignis. „Wer verzapft denn hier so einen Blödsinn... , ich glaub’ mein Schwein pfeift!“; „Ich glaube, mein Schwein pfeift! Das kann ja wohl nicht wahr sein!“

(6) Ich glaub, mein Hamster bohnert! Ausruf der Überraschung/Verwunderung/Verärgerung/des Erstaunens.

(7) Ich glaub, mich streift ein Bus! Ausruf der Verwunderung/Überraschung/ des Erstaunens/der Verärgerung „Ich glaub’, mich streift ein Bus - du hast ja tatsächlich aufgeräumt!“

(8) Ich denk/glaub, mich trifft der Schlag! Sagt man bei einer unangenehmen Überraschung.

Diese Bespiele passen nicht nur der Form nach ins Paradigma, auch der Inhalt der Nebensätze spiegelt die Inhalte von (1-4) wider. Die Wahrnehmung sich eigenartig verhaltender Tiere sind ein gern benutztes Ingredienz (5-6), und die Empfindung eines (plötzlichen) Schlages, wie in Mich tritt ein Pferd wird auch in (7-8) zum Ausdruck gebracht. Das Gefühl, sich plötzlich, unerwartet, in einer falschen Umgebung zu befinden, wie sie in Ich denk, ich steh im Wald zum Ausdruck gebracht wird, findet sich auch in (9):

(9) Denken/glauben, im falschen Film (gelandet) zu sein. Mit einer ungewöhnlichen/unangenehmen/unverständlichen Situation konfrontiert werden; das Gefühl haben, fehl am Platz zu sein.

In Redensarten gibt es weitere vier Wendungen, die die eigene Wahrnehmung und Kognition thematisieren. Zwar folgt der Inhalt des Nebensatzes nicht dem Muster der Absurdität, das bei den anderen Wendungen vorherrscht, aber Überraschung kommt zum Ausdruck, diesmal indirekt, durch Zweifel an der eigenen Wahrnehmung (,kann nicht wahr sein“): 
(10) Ich denke/denk/glaube/glaub, ich sehe/seh nicht recht. Ich bin völlig (meist: unangenehm) überrascht. „Als ich in den Spiegel geschaut hab, dachte ich, ich seh nicht recht: Meine Augen waren so gequollen, dass ich sie kaum öffnen konnte und mein ganzes Gesicht hatte rote Flecken.“

(11) Ich denke/denk/glaube/glaub, ich höre/hör nicht recht. Ich bin völlig (meist: unangenehm) überrascht. „Mein Kollege meinte, er zahlt 600 Euro an Steuer für das Auto. Ich dachte, ich hör nicht recht“.

(12) (Ich glaub',) ich werd' nicht mehr/wieder! Ausruf des Erstaunens/der Überraschung/des Erschreckens/der Bestürzung

(13) Ich glaub, ich lüge! Ausdruck der Verärgerung/des Erstaunens

Diese Auflistung erhebt keinen Anspruch auf Vollständigkeit. Eine weitere Suche im Internet ergibt z.B. Ich glaub, es hackt! und Ich glaub, ich spinne! Auch hier tauchen wieder die Themen des harten Schlages und der Zweifel an dem eigenen Verstand auf. Wir können abschließend feststellen, dass es sich um ein offenes Paradigma handelt, wobei neue Wendungen von den schon vorhandenen inspiriert werden, aber zugleich sprachliche Kreativität aufweisen.

\section{Abwandlungen}

Wenn wir uns die Wendungen (1-4) in Duden 11 noch einmal anschauen, fallen neben dem gleichen Format auch kleine Varianten auf. In (1) wird die Wendung im Präsens aufgeführt, aber der Textbeleg fängt mit Ich dachte an, Präteritum also. In (2) werden knutschen und küssen als gleichwertige Varianten spezifiziert. Und in (3) steht der Matrixsatz in Klammern. In diesem Abschnitt werden wir uns weitere Abwandlungsmöglichkeiten anschauen.

\subsection{Abwandlungen im Nebensatz}

„Das Bedürfnis, Konstruktionen mit abgeschwächter 'verschlissener' Expressivität durch solche mit stärkerer Expressivität zu ersetzen, ist eine Quelle ständiger Bereicherung des Bestandes an phraseologischen Synonymen“ (Fleischer 1997: 178). Diese Einsicht findet volle Bestätigung in dem Mich-tritt-ein-Pferd-Paradigma, sowohl in der Zahl der Ausdrücke an sich als auch in den kleineren Varianten zu den einzelnen Wendungen. 
In Redensarten findet sich beim Eintrag Ich glaub/denk, mich tritt ein Pferd! eine längere Erläuterung, in der verschiedene andere Wendungen und Varianten erwähnt werden, wie Haifisch als Alternative zu Elch, D-Zug als Alternative zu Bus. Die Wendung Ich glaub', mein Pferd schielt!, die dort auch aufgeführt wird, könnte man als Variante von Ich glaub, mein Schwein pfeift auffassen. Weitere Abwandlungen, die ein eigenartiges Verhalten eines Tieres thematisieren, sind folgende:

(14) ich glaub, mein Hund spielt Halma. ${ }^{4}$

(15) Ich glaube, mich rammt ein Rotkehlchen. ${ }^{5}$

Im folgenden Beispiel klingt die mich-streift-ein-Bus-Wendung an. Die Variante Glotze wird hier wahrscheinlich wortspielerisch durch das im Kontext anwesende Bildschirmen getriggert.

(16) Mobile Werbung in den USA: Neuartige Bus-Außenkarosserie aus LCDBildschirmen.... Ich glaub', mich streift 'ne Glotze... (1. Januar 2014). ${ }^{6}$

Unzweifelhaft kann man weitere kreative Abwandlungen finden. Wie Fleischer betont, ist es expressiven Wendungen inhärent, dass sie sich erneuern.

Eine Konstante im Nebensatz ist die Wortstellung: immer SVO. Mit anderen Worten, die unterordnende Konjunktion dass, die SOV erzwingen würde, ist systematisch abwesend. Ich glaub, dass mich ein Elch küsst würde kein Muttersprachler als „korrekt“ ansehen. Das deutet darauf hin, dass das Matrixverb nicht im wörtlichen Sinne von „an etwas glauben“ oder „einen Denkakt vollziehen“ verstanden werden sollte, sondern in einem abstrakteren Sinne als „epistemic qualifier“ (Imo 2011), der zum Ausdruck bringt, dass ein Interpretationsakt vorliegt, der zu dem im Nebensatz spezifizierten absurden Ergebnis kommt, das der Sprecher eigentlich nicht für möglich hält.

\footnotetext{
4 www.swr.de/blog/1000antworten/antwort/2540/warum-heisst-es-ich-glaube-mein-schweinpfeift (Stand: 25.7.2017).

5 www.abendblatt.de/ratgeber/wissen/article119412122/Woher-stammt-der-Ausdruck-Ichglaube-mein-Hamster-bohnert.html (Stand: 25.7.2017).

$6 \mathrm{https}$ //twitter.com/search?q=ich\%20glaub\%20mich\%20streift\%20ne\%20Glotze\&src=typd (Stand: 25.7.2017).
} 


\subsection{Abwandlungen im Matrixsatz}

Als erste Variante im Matrixsatz ist die zwischen glauben und denken zu erwähnen. Obwohl Duden 11 nur Varianten mit denken bietet, kommen in Redensarten und an anderen Stellen beide Varianten vor. Sowohl denken als auch glauben sind mentale Verben, die einen bestimmten Grad der Unsicherheit implizieren, im Gegensatz zu wissen, das Sicherheit ausdrückt. Denken bezieht sich auf den mentalen Prozess, der zu dem im Nebensatz erwähnten kognitiven Resultat führt, während glauben eher auf das Resultat selbst fokussiert. Aber dieser kleine Unterschied macht für den Gebrauch im Kontext der mich-tritt-ein-Pferd-Wendungen nichts aus. Beide Varianten bieten dem Sprecher die Möglichkeit, anzudeuten, dass er seine eigene Interpretation nicht glauben kann. Einmal fand ich scheinen als Matrixverb, Mir scheint, mein Hamster bohnert, was auch in das Paradigma der Unsicherheitsausdrücke passt. ${ }^{7}$

Auch quantitativ lässt sich nicht leicht ein Gebrauchsunterschied zwischen glauben und denken in diesem Kontext finden. Die Zahl der Treffer für Ich glaub' mich tritt ein Pferd ist 159.000 und für die Variante mit denken 37.400, aber dieser Unterschied kann mit der unterschiedlichen Popularität der beiden Filme zu tun haben. Bei Ich glaub'/denk' mich laust der Affe gibt es 64.400 Treffer für glauben und 36.000 für denken (26.7.2017).

Der Matrixsatz wird nur selten weggelassen. Es fällt aber auf, dass vor allem Mich laust der Affe regelmäßig ohne Matrixsatz vorkommt, vgl. auch den Buchtitel (Dr. Wort, 2012). Redensarten klammert auch bei Ich glaub, ich werd' nicht mehr/wieder den Matrixsatz ein. Dahingegen habe ich zu Mein Hamster bohnert kein einziges Beispiel ohne Matrixsatz finden können. Warum Mich laust der Affe und Ich werd' nicht mehr/wieder sich als Ausnahmen verhalten können, ist mir nicht klar.

Imo (2011) hat gezeigt, dass ich glaub sich in bestimmten Kontexten zu einem „epistemic qualifier“ entwickelt hat, der dann auch parenthetisch in der Satzmitte oder am Satzende auftreten kann (z.B.: Das wär so schwierig geworden glaub ich, Imo 2011: 174). Obwohl ich glaub, und auch ich denk, in den hier besprochenen Wendungen durchaus als „epistemic qualifier“ charakterisiert werden kann (siehe auch den letzten Satz in 3.1), ist diese positionelle Abwandlung beim Gebrauch im Kontext des Paradigmas nicht möglich (\#Mich tritt ein Pferd glaub ich). Die feste Reihenfolge von Matrix- und Nebensatz macht ikonischen Sinn: Der Sprecher gibt mit dem Matrixsatz an, dass er vor einer Interpretationsaufgabe steht, die im Nebensatz seine absurde Auflösung findet.

7 www.helmut-froehlich.de/Musik/Roski/Roski-Texte_chronologisch.pdf (Stand: 27.7.2017). 
Die Form des Matrixsatzes ist ziemlich fest: 1. Person Subjekt + finites Verb. Ein Adverb kann hinzugefügt werden (Ich dachte echt, ich steh im Wald; dachte auch mich tritt ein Pferd, mit Weglassung des Subjektes; beide Beispiele aus Twitter, 26.7.2017). Beispiele in der 3. Person sind selten, aber sie kommen vor: Doch was er dann sehen muss, er glaubt für wahr ihn streift ein Bus. ${ }^{8}$

Präsens ist die übliche Zeitform, aber es ist nicht schwierig, im Internet Beispiele im Präteritum zu finden, auf jeden Fall für dachte, vgl. folgende Beispiele:

(17) Der Spruch, „die Schule sollte meinen Namen bekommen“ da dachte ich, mein Hamster bohnert...weswegen? Soll jetzt jeder der von der Schule flog, zur Belohnung den Namen geben dürfen? ${ }^{9}$

(18) Ich dachte mein Schwein pfeift und mein Hamster bohnert. Manche Menschen sind so verblendet. Da finden die es doch tatsächlich toll, nur 500 kcal am Tag hauptsächlich Obst und Gemüse (wenigstens etwas) zu sich zu nehmen. ${ }^{10}$

(19) Ich dachte mich streift ein Bus, als ein Kölner Freund da ernsthaft eine Feminismusdebatte anzetteln wollte. ${ }^{11}$

Eine Internetsuche nach Wendungen mit glaubte als Präteritum ergab dahingegen nur ein paar Beispiele:

(20) Meine Frau hat mir die von mir gekochte Lasagne gestern nachgeworfen, weil sie noch halb gefroren war. Ich glaubte, mich tritt ein Pferd!!!12

(21) Ich bestellte vorab schon mal einen kleinen Prosecco. Dann ging die Tür auf, ich glaubte mich tritt ein Pferd. Nicht wieder zu erkennen, es war Anny. Die Haare offen, ohne Brille, eine weiße Seidenbluse, ein Tuch um den Hals geschlungen. ${ }^{13}$

\footnotetext{
8 http://docplayer.org/28666633-2006-i-r-s-ryffi-fw-ffif-f-ffi-s-i-f-t-fl-jd-w-l-r-1-trir1-li-r-s-r-dj-nlf-fr-t.html (Stand: 26.7.2017).

9 www.facebook.com/meinRTL/photos/a.317833411562117.86456.179133132098813/937317589 613693/?type=1\&theater (Stand: 26.7.2017).

10 http://fetterblog.fettloeserin.de/2014/09/22/genau-fur-sowas (Stand: 26.7.2017).

11 https://twitter.com/CaeVye/status/860638945029349376 (Stand: 26.7.2017).

$12 \mathrm{https} / /$ twitter.com/ulumbamulumba/status/302295812262748160 (Stand: 27.7.2017).

13 Voss, Michael (2011): Hi Liebling - ja, mein Schatz!. Hamburg: BoD Verlag. 78
} 
Beispiele im Perfekt sind noch seltener, aber auch die gibt es, vgl:

(22) Schon die Startsequenz ist der Hammer, ich habe geglaubt mich tritt ein Pferd... ach nö ein Esel. ${ }^{14}$

(23) Als du in mein Leben tratst - Arthur... ich hab geglaubt, mich tritt ein Pferd! $!^{15}$

Die phonologische Variation mit und ohne Flexions-e ist beim Verb nicht spezifisch für das Paradigma, sondern ein allgemeines Phänomen in der gesprochenen Sprache. Im Duden 11 und auch in Redensarten wird als stilistisches Merkmal der Wendungen oft „salopp“ oder „scherzhaft“ hinzugefügt. Zu diesem Stil passt die Weglassung der Endung natürlich gut. In der schriftlichen Wiedergabe der Wendungen wird die Weglassung oft mit Apostroph (glaub', denk') wiedergegeben.

\section{5 Überraschung, Exklamativ und Mirativ}

In der Konstruktionsgrammatik wird keine scharfe Grenze zwischen Lexikon, Grammatik und Phraseologie gezogen. Es ist also angebracht, sich die Frage zu stellen, ob bestimmte allgemeine syntaktische oder semantische Begriffe Relevanz für das Mich-tritt-ein-Pferd-Paradigma haben.

Dalmas (2004: 74) hat einige allgemeine Merkmale für Exklamationen formuliert: „Exklamativäußerungen sind in erster Linie sprecher-zentriert. Sie drücken eine affektiv-emotionale Einstellung des Sprechers aus, der auf einen normabweichenden und von ihm nicht erwarteten Sachverhalt verbalkommentierend reagiert.“ Diese Charakterisierung trifft sicher auch für die Wendungen im Paradigma zu. Bei gesprochenen Beispielen nimmt die Intonation in Ich glaub/denk typischerweise einen ruhigen Anlauf, um im Nebensatz intensiv zu enden. In der schriftlichen Wiedergabe wird oft ein Ausrufezeichen hinzugefügt.

„Normale“ Exklamationen setzen typischerweise Faktizität voraus: Dasjenige, was den Sprecher überrascht, wird als gegeben, wahr, vorausgesetzt. Das ist bei den Mich-tritt-ein-Pferd-Wendungen auch der Fall, nämlich für den im Kontext erwähnten Anlass. Ausdruck der Überraschung wird sowohl in Duden 11 als auch in Redensarten bei den meisten Wendungen im Mich-tritt-ein-Pferd-Para-

14 http://forums-de.ubi.com/archive/index.php/t-36451.html (Stand: 26.7.2017). 15 www.markus-fenner.de/arthur.html (Stand: 26.7.2017). 
digma als zentrale Bedeutungskomponente angegeben. Auch andere Gefühle können mitschwingen: Frustration, Empörung, Entrüstung. Meistens geht es also um eine unangenehme Überraschung, aber sie kann auch angenehm sein (wie im Beispiel bei Wendung 2).

„Mirativ“ ist eine strengere Kategorie als Exklamativ (vgl. die Diskussion in Olbertz 2012), die vielleicht auch auf das Paradigma zutrifft, vgl. DeLancey (1997: 33): „The fundamental function of the [mirativity] category is to mark sentences which report information which is new or surprising to the speaker“. „Mirativ“ wird primär als eine Bedeutungskategorie angesehen, die in grammatischen Systemen bestimmter Sprachen morphologisch verankert ist. Sprachen ohne Mirativmorphologie bringen Überraschung durch andere Mittel wie Intonation oder spezielle Konstruktionen zum Ausdruck. In der Literatur zu Mirativität wird diskutiert, ob die Kategorie „Mirativität“ auf morphologische Ausdrucksmittel beschränkt werden sollte oder ob der Begriff im erweiterten Sinn benutzt werden darf, also für alle sprachlichen Mittel, die Überraschung ausdrücken. Aikhenvald hat mehrmals für die erste Option plädiert, vgl. Aikhenvald (2012: 475): „[I]t would not be appropriate to extend mirative, or the grammatical category of 'expectation of knowledge', to lexical means.“ Aber viele andere Autoren benutzen den Begriff im erweiterten Sinn, und dann spricht nichts dagegen, dem Paradigma Mirativbedeutung zuzuweisen.

Ein zusätzliches Kriterium für den Mirativstatus einer Äußerung ist, dass es sich um eine Wahrnehmung handeln muss, die im Hier-und-jetzt stattfindet, von Rett/Murray (2013: 456) als „recency restriction“ angedeutet. Diese Bedingung trifft beim Paradigma zu, mit dem Unterschied, dass die neue Information nicht in der Äußerung selbst vorkommt, sondern im Kontext, direkt vor oder nach der mirativen Äußerung. Mit dieser Einschränkung kann man sagen, dass die Äußerung, in der der Anlass formuliert wird, zusammen mit der Mich-tritt-ein-PferdÄußerung eine „diskursive“ Mirativkonstruktion bildet.

\section{Fazit}

In diesem Beitrag wurde gezeigt, dass die deutsche Sprache über ein Paradigma von Wendungen verfügt, die allesamt starke Überraschung ausdrücken. Die morphologische und syntaktische Flexibilität der Wendungen hält sich in Grenzen, aber lexikalisch gibt es immer wieder neue kreative Abwandlungen, was typisch für ein expressives Paradigma ist. Die Expressivität betrifft primär das Gefühl der Überraschung, weshalb ich der Meinung bin, dass die semantische Kategorie „mirativ“ angebracht ist. Das, worauf sich die Wendung bezieht, der 
Anlass zur expressiven Äußerung, befindet sich im textuellen oder situationellen Kontext.

In diesem Beitrag wurden Beispiele illustrativ herangezogen. In einem nächsten Forschungsschritt wäre es wünschenswert, Korpora vollständig auszuwerten, damit das Vorkommen der Wendungen in verschiedenen Stilen und Genres genauer geklärt wird. Auch wäre noch zu klären, wie „dramatisch“ der Anlass sein muss, damit der Gebrauch einer Wendung aus dem Paradigma nicht übertrieben wirkt. Ursprünglich hatte ich Martine schon 65? Ich denk, mich tritt ein Pferd! als Titel dieses Beitrags gewählt. Ein Muttersprachler gab aber zu bedenken, dass sich das irgendwie nicht richtig anhört, der Anlass sollte „eigenartiger“ sein, damit es mit einer Wendung aus dem Paradigma kommentiert werden kann. Ich bin gespannt, was eine systematische „Anlassstudie“ dazu sagen würde.

\section{Literatur}

Aikhenvald, Alexandra (2012): The essence of mirativity. In: Linguistic Typology 16, 3. 435-485.

Dalmas, Martine (2004): Wenn einer ruft ... Ausrufe, Aufrufe und dergleichen. Abgrenzung aufgrund diskursiver Haltung. In: Krause, Maxi/Ruge, Nikolaus (Hgg.): Das war echt spitze! Zur Exklamation im heutigen Deutsch. Tübingen: Stauffenburg. 67-76.

DeLancey, Scott (1997): Mirativity: The grammatical marking of unexpected information. In: Linguistic Typology 1, 1. 33-52.

Dr. Wort (2012): Mich laust der Affe. Neues aus der Welt der Redewendungen. Reinbek: Rowohlt.

Duden (2002): Duden 11: Redewendungen. Wörterbuch der deutschen Idiomatik. 2., neu bearb. u. aktual. Aufl. Mannheim u.a.: Dudenverlag.

Fleischer, Wolfgang (1997): Phraseologie der deutschen Gegenwartssprache. 2., durchges. u. erg. Aufl. Tübingen: Niemeyer.

Imo, Wolfgang (2011): Clines of subordination - constructions with the German 'complementtaking predicate' glauben. In: Laury, Ritva/Suzuki, Ryoko (Hgg.): Subordination in conversation. A cross-linguistic perspective. Amsterdam: Benjamins. 165-190.

Olbertz, Hella (2012): The place of exclamatives and miratives in grammar. A functional discourse grammar view. Linguistica 8, 1. 76-98.

Rett, Jessica/Murray, Sarah E. (2013): A semantic account of mirative evidentials. In: Proceedings of SALT 23. 453-472. 
